\title{
Just in time: defining historical chronographics
}

\author{
Stephen Boyd Davis \\ Lansdown Centre \\ Middlesex University \\ Cat Hill, Barnet, Herts \\ EN4 8HT, UK \\ s.boyd-davis@mdx.ac.uk
}

\author{
Emma Bevan \\ Lansdown Centre \\ Middlesex University \\ Cat Hill, Barnet, Herts \\ EN4 8HT, UK \\ emmalucybevan@googlemail.com
}

\author{
Aleksei Kudikov \\ Lansdown Centre \\ Middlesex University \\ Cat Hill, Barnet, Herts \\ EN4 8HT, UK \\ aleksei@hotmail.co.uk
}

\begin{abstract}
The paper is historical in two respects, both concerned with visual representations of past time. Its first purpose is to enquire how visual representations of historical time can be used to bring out patterns in a museum collection. A case study is presented of the visualisation of data with sufficient subtlety to be useful to historians and curators. Such a visual analytics approach raises questions about the proper representation of time and of objects and events within it. It is argued that such chronographics can support both an externalised, objectivising point of view from 'outside' time and one which is immersive and gives a sense of the historic moment. These modes are set in their own historical context through original historical research, highlighting the shift to an Enlightenment view of time as a uniform container for events. This in turn prompts new ways of thinking about chronological visualisation, in particular the separation of the 'ideal' image of time from contingent, temporary rendered views.
\end{abstract}

Timeline. Chronology. Chronographics. Time. Visualisation. History. Museums.

\section{INTRODUCTION}

The paper focuses on the 'shape' of time in digital visualisations of history, and considers the positioning of objects and events in documents such as timelines. We exemplify these using a recent prototype for a museum. Working on it caused us to reflect on the mapping of time to visual space, which we then pursued by researching the early days of modern visualisation, which in turn clarified our thinking about the future design of such chronographic forms in digital media.

\section{A TIMELINE OF MUSEUM OBJECTS}

Historical Interactive Timeline (HiT) is a prototype chronographic interface to a database at the Museum of Domestic Design and Architecture (MoDA) at Middlesex University in north London, UK. MoDA holds collections from the late nineteenth to mid twentieth century mainly concerned with the visual culture of everyday domestic interiors. Its core collection comprises Silver Studio wallpaper, fabric and designs from 1880 onwards. It designed for clients including Liberty and London Underground until 1963.

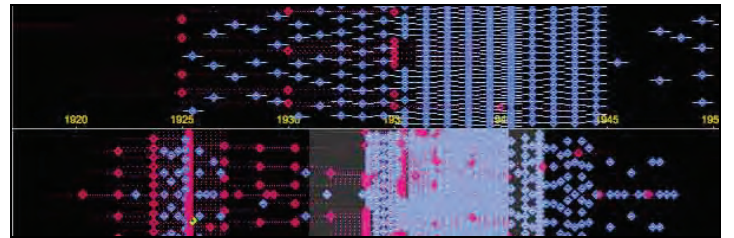

Figure 1: Bevan and Kudikov: HiT interface for MoDA (detail). At top, part of the main view spanning 1910s to 1950; below, the comprehensive view of the entire dataset

MoDA uses a single database for collections management and as its source of public information such as its website. Curators access the data via a basic textual interface and can only view multiple records as series of lists. The web interface offers similar lists plus a simple tabular graphic view. One cannot organise items in relation to one another, place the collection in the context of time, or at a glance see when pieces were produced. Having a chronographic or timelinebased presentation would give curators, historians and museum visitors alike a sense of the physical size of the collection, an idea of its breadth over time, and enable them to view images in these contexts and to discover periods when production was sparse or intense. They could investigate when floral patterns were produced in greatest number, or find the most popular production 
technique during the Art Deco period. The dataset has approximately 10,500 records of 160 fields: for our prototype we worked with one fifth of these. Figure 1 shows a detail of our display in which the lower band offers a miniature view of the entire dataset: the patterning and altered densities over time which we sought to reveal are clearly visible.

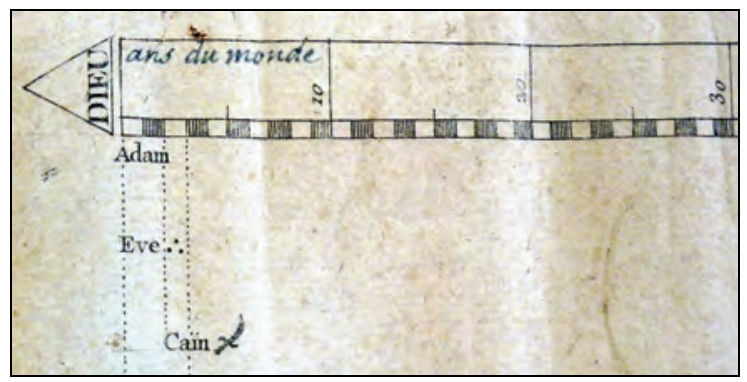

Figure 2: Barbeu-Dubourg: chronographic chart 1753 (detail). The beginning of time

\section{CHRONOGRAPHICS}

Considering the mapping of events and objects to time led us to question some of our basic assumptions. In particular, we became fascinated by the very notion of positioning objects in a fixed, constant timeframe.

Clearly there are many ways to visualise time. Some such as the dials of clocks emphasise its cyclical qualities; others such as diaries and calendars are more linear. In visualising history, strongly linear presentations tend to predominate. Nevertheless there is still scope for considerable variation. For example, events may be packed closely, they may be positioned arithmetically along a uniform scale or on a non-linear scale or, as often with paper timelines, using a scale that is altered pragmatically to accommodate unevenness in the data. These representations of history have a history of their own, which can cast light on some important issues: this paper is therefore also an argument for the value of historical perspectives on modern visualisation.

\subsection{Two historic examples}

In the mid 18th century two revolutionary charts appeared. In 1753 in Paris, Jacques BarbeuDubourg (1709-1779) created a chart 16.5 metres long plotting all history from the Creation to his own date on a uniform timescale (Ferguson, 1991) (Figure 2). Also using a uniform scale, in 1765 Joseph Priestley (1733-1804) for the first time represented the duration of individuals lives using printed lines (Twyman, 1986) (Figure 3). Though these objects are fascinating in themselves, it is their conceptual basis that interests us here.

\subsection{From chronology to chronography}

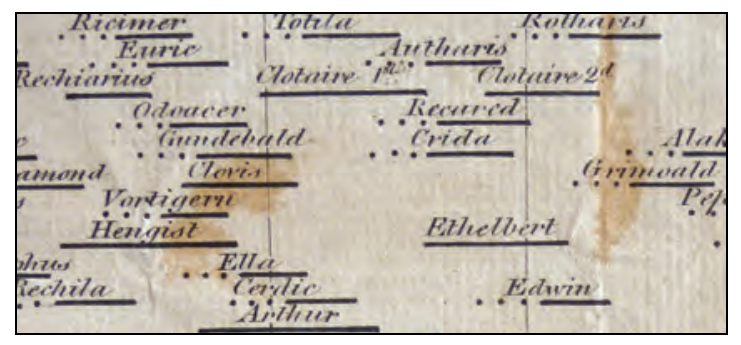

Figure 3: Priestley: chronographic chart 1765 (detail). Priestley invented the use of a line to represent a

lifespan. He also used dots to represent uncertainty

Dates might seem merely a routine technical underpinning of history, but in the past chronology was a serious study in its own right. Feeney (2007) records the practice in classical times. In the 18th century, 'history' had connotations of narrative and story, to which chronology brought rigour: it brought (various authors argued) meaning, vividness, memorability, an evidential basis, and a unifying framework. A writer as august as Locke (1693) considered chronology necessary to give form to history, making it more memorable and more productive of moral lessons.

\subsubsection{The rise of the visual}

A recurrent visual motif, dating back at least to the early 17 th century, is that chronology and geography are the 'two eyes of history' (Purchas, 1614, p. 613). In the Enlightenment, appropriately, the metaphor of illumination is common; historical events are 'cover'd with a Cloud of Darkness [...] till the Lamp of Historical Chronology has enlighten'd them' (Blair 1754); without it we will 'wander in the dark, and be always at a loss' (Emerson 1770). Mid-century, these metaphors of the visual are actualised: Twyman (1990) describes how relationships between sets of information are made visually explicit, including not only the timelines discussed here, but Lambert's line graphs (176070s) and Playfair's 'lineal arithmetic' (1780s).

Chronology becomes chronography when duration is mapped to spatial dimension. Pre-18th century chronologies by contrast almost always packed each entry close upon the previous one; as Blair (1754) complained, they have 'all of them made one great and fundamental Mistake [...] the contracting History into as little Room as they could...' However, in the early 17th century Helvicus (1609) created tables using equal space (in his case equal pages) for equal time. Now the reader could tell at a glance the interval between two dates: instead of having to read and compute the interval, the user could see it. Nevertheless, it would take another 140 years for historic time to be mapped arithmetically to space on a continuous 
substrate, rather than table-wise in a series of pages. The innovator was Barbeu-Dubourg.

For him, geography is not merely parallel with chronology as in the 'two eyes' metaphor but the inspiration for change: 'The study of Geography is pleasing, easy, attractive [...] This is not how it is with Chronology, which is a dry form of study, laborious, unforgiving, offering nothing to the mind but repellent dates, a prodigious accumulation of numbers which burden the memory, are difficult to lodge in the mind and escape thence all too easily' (Barbeu-Dubourg, 1753, p. 5). His solution is to make time like geography: 'May not duration be imitated and represented as effectively to the senses, as distinctly as space, and may not intervals of time be as easily counted in degrees?' $\mathrm{He}$ insists that 'charts of time can and should be constructed all at the same scale, with a constant representation of the years' - because that way we can directly compare intervals. But before time could be conceived as mappable to a uniform space in this way, it had itself to be perceived as a uniform measure. To do so might seem simply natural, but several prior concepts were necessary.

\subsubsection{The origins of uniform time}

A necessary antecedent to chronography proper is Newton's 'absolute, true, and mathematical' time as the measure of all events (Newton 1687). It is hard for us now to capture the excitement of d'Alembert (1753), quoting Newton at the beginning of Chronologie in Diderot's Encyclopaedia, 'All things are placed in time as to order of succession; and in space as to order of situation,' yet such a view of time as analogous to spatial measurement was an innovation. Nevertheless as Rosenberg (2007) points out, even Newton's chronologies were not actually graphical: they were lists like those of his predecessors. For the basis of this innovation we have to turn to Descartes.

Descartes' writings, particularly the Compendium Musicae of 1619, reveal a fixation with the ideas of clear representation, of grasping magnitudes at a glance, and specifically of line-lengths which correspond to number (Gaukroger, 1995). In Rule 24 of the posthumous Regulae of 1684 we find that 'the infinite multiplicity of figures [i.e. graphical forms] is sufficient for the expression of all the differences [i.e. any variables] in perceptible things'. Descartes describes the number-line: 'if addition or subtraction is to be used, we conceive the subject in the form of a line, or in the form of an extended magnitude in which length alone is to be considered.' This is echoed later not only by Barbeu-Dubourg's argument for measuring time like distance but even more exactly by Priestley's explanation that time 'admits of a natural and easy representation in our minds by the idea of a measurable space, and particularly that of a line; which, like time, may be extended in length, without giving any idea of breadth or thickness.' By the late 18th century William Playfair (1759-1823) the inventor of the line chart would recall his mathematician brother John teaching him that 'whatever can be expressed in numbers may be represented in lines,' (Costigan-Eaves \& Macdonald-Ross, 1990, p. 324). Before Descartes, this idea could not have been thought.

There is mutual influence here: once a quantity in the mind can be externalised as line-length, the notion of time-as-dimension itself can become internalised. Feeney (2007, p. 8) remarks on the near-impossibility of our recovering in imagination the character of events before the advent of our 'universal numerical grid' for history, when time was, in Poole's words 'an uneven succession of periods of different qualities, a cluster of high points and low, rather than a steady stream of being' (1998, p. 21). Gell (1992, p. 23 passim) warns that to even refer to a culture's 'model of time' is to beg the question: a society may have a model of the relationship between events, between now and the past, between the past and the future, etc., but not necessarily have a model of time at all.

In considering our pioneers' excitement about uniformity, contemporary visual culture had a possible role. Tull's improved design of seed drill, though not widely adopted, would have been familiar in 'improving' circles: hand-broadcast seed replaced by equidistant straight lines of plants. The century also saw the growth of urban terraces in improved cities like Bath and Edinburgh, distinctive for their simple repetition and the equality of every unit in the façade. Even pavements, until mideighteenth century in Britain the responsibility of individual householders and therefore of different heights, materials and quality, began to be replaced by uniform paving funded through local taxation. Such progressive visual uniformity surely must have influenced forward-thinking intellectuals.

\subsubsection{Branches, chains, streams, rivers, arrows}

Simplistic distinctions have been made between cultures such as the ancient Greeks, said to have a circular conception of time, and the linear teleological time of Judaism and Christianity. Feeney (2007, p. 3) points out that we ourselves habitually switch between models depending on context. The 18th century balanced metaphors of time's cycle and time's arrow (Gould, 1987), but most modern timelines, including our own HiT, are linear. They are direct descendants of BarbeuDubourg and Priestley's models.

When using a physical substrate such as paper, a linear model is partly just a matter of convenience. But another motive for both pioneers seems to have been their exceptional optimism about 
progress: Barbeu-Dubourg's Petit Code de la Raison Humaine of 1789 seems to look forward to the imminent reform of society through reason, and Priestley (1764, p. 25) explicitly projects the progress of his own time into the future:

What a figure must science make, advancing as it now does, at the end of as many centuries as have elapsed since the Augustan age!

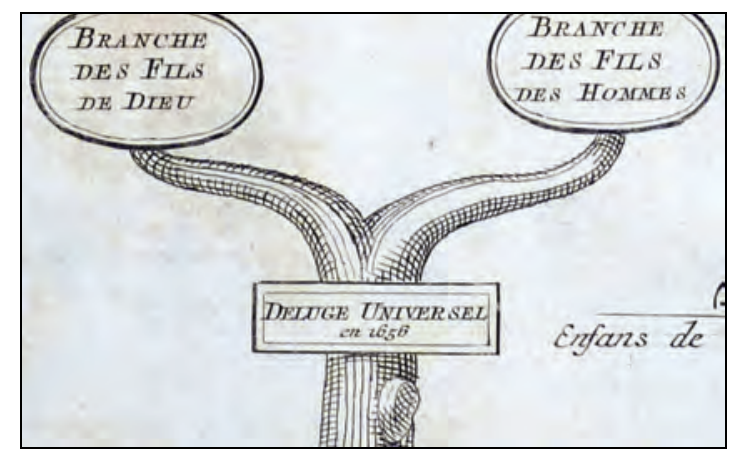

Figure 5: Châtelain, 1721: Chain of Sacred History (detail) from the Atlas Historique. Time as branching plant. Collection: Stephen Boyd Davis

Figure 5 illustrates a different graphical metaphor for historical events, the branching plant, complete with a severed offshoot - though the work's title uses the more linear notion of a chain. As in genealogy, the aim here is not primarily to show positions in time - the topography of events - as the relations between them - their topology (Rosenberg 2007 provides an extended discussion of such metaphors). Priestley is fond of the river: for him universities are pools of stagnant water compared with the Dissenting academies which are 'like rivers which taking their natural course fertilise a whole country' (Priestley 1787 quoted in Sheps 1999, p. 141). Though he avoided any figurative elements in his charts of biography and history, the metaphor of the river is never far away. In his explanatory Description (1764) he explicitly invokes rivers, citing their lack of beginning and end and likening the lives of men to 'so many small straws swimming on the surface' (p. 24). His chart of biography bears the motto fluminis ritu feruntur, evoking Horace's advice to Maecenas to maintain his own position while the world flows around him like a river. In either case, such a river is more or less formless. Quite unlike the branching organism of the genealogy or Châtelain's Chaine, it has no internal structures. Though both Barbeu-Dubourg and Priestley impose some order on their streams using categories on the non-time axis (nations in Barbeu-Dubourg's case and types of individual in Priestley's), there is a distinct lack of structural or relational information. Nevertheless, the apparently simple river metaphor affords two alternative interpretations.

\subsubsection{Observation and immersion}

Priestley's river of time metaphor takes two forms. In the Description, we seem to stand on the riverbank watching time's flow from without, while with the Horace quotation Priestley puts man within the river as the waters swirl around him. In relation to the first usage, an obvious aspect of the chronographics we have been discussing is that they do indeed provide an overview, seeming to chime with everyday metaphors of standing back in order to get situations in perspective, being too close to an issue, being unable to see the wood for the trees, and so forth. A less obvious potentiality is to create a sense of immersion in the historic moment. Priestley describes this as follows:

It is a peculiar kind of pleasure we receive, from such a view as this chart exhibits, of a great man, such as Sir Isaac Newton, seated, as it were, in the circle of his friends and illustrious cotemporaries. We see at once with whom he was capable of holding conversation, and in a manner (from the distinct view of their respective ages) upon what terms they might converse.

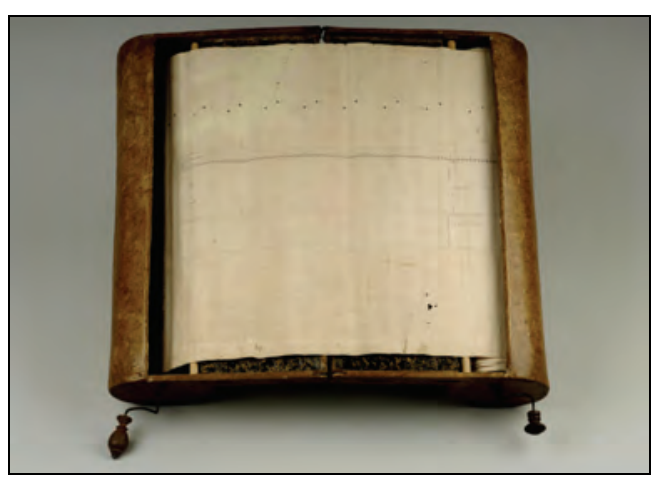

Figure 6: Barbeu-Dubourg: chronographic chart 1753. The 16.5 metre long scrolled timeline in its machine. Princeton University Library. With permission

Priestley's time charts are static, so the flow of his river of time is arrested as in a snapshot. However, immersion is a strong feature of an extraordinary creation by Barbeu-Dubourg, enthusiastically described in great detail by Diderot (1753) in the Encyclopédie. He inserted his continuous paper roll of time into a machine chronographique, with small crank handles to wind history back and forth: about 150 years are visible at any moment (Figure 6). He calls his invention:

a moving, living tableau, through which pass in review all the ages of the world, where each famous figure steps forth in his rank with the attributes belonging to him, where each Prince is surrounded by his contemporaries and occupies the scene for more or less time according to the duration of his role, where the rise and fall of Empires are acted out in visible form.. 
Here the sense of history as immersive experience is uppermost.

Like Priestley's or Barbeu-Dubourg's, the visualisations we construct may favour the observational or immersive mode. One factor that differentiates these modes is perceived distance. Seeing the full scope of a comprehensive data-set, patterns emerge for the observer who stands outside time; but when the user moves closer and studies individuals in their context, instead a sense of immersion is promoted. This is an underemphasised aspect of the relationship between focus and context discussed at length, for example, in Card et al. (1999).

\section{ISSUES IN VISUALISING TIME}

Priestley makes a superb case for visualisation (Priestley, 1764, p. 10). He uses the example of trying to figure out the relationship between the lives of five historical figures: he allows his reader to experience the difficulty of answering questions about their relative dates before directing them to look at his chart:

as soon as you have found the names, you see at one glance, without the help of Arithmetic, or even of words, and in the most clear and perfect manner possible, the relation of these lives to one another.

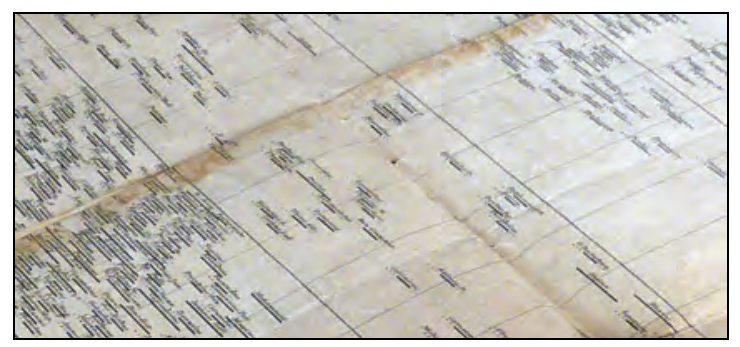

Figure 7: Priestley, 1765: Chart of Biography (detail). Clusters and absences become clear at a distance

His explanatory book, though only twenty-five pages long, offers over fifty thoughts relevant to visualisation on themes including perception, visual metaphors, salience, recall, data integrity, categorisation and problems of objectivity. He was the first and for many years the only chronographer to care about representing uncertainty, something we carried through in HiT, always indicating the accuracy of our dates by a span rather than a point.

Priestley's understanding of the benefits of a truly spatial layout is striking: 'the thin and void places in the chart are, in fact, not less instructive than the most crowded' (p. 24-5). Absence, he realises, is a form of information, and is most easily brought out by a constant scale. Such patterns are visible even at the reduced scale of Figure 7 , as we noted in
HiT (Figure 1). He criticises a recent French predecessor to his own chart (not BarbeuDubourg's) for its non-linear design. The most impressive point is his reason: 'the notice which is given of this change [in scale] is not sufficient to correct the error of the imagination' (p. 8) - he realises that first impressions resist subsequent attempts to intellectually reinterpret what has been seen. These and his other observations are remarkable at the dawn of modern visualisation. It has to be admitted that Barbeu-Dubourg's remarks by comparison are more reminiscent of the hyperbole around multimedia in the 1980s and 90s: they mainly comprise claims that data will be effortlessly imbibed and recalled - and that the whole process will be enjoyable.

Despite the cogency of many of Priestley's observations, he and Barbeu-Dubourg present onesided arguments for uniform timescales. From our greater distance it is worth considering the counterarguments they chose to ignore.

\subsubsection{The problem of data density}

In 1838 a second, posthumous edition of BarbeuDubourg's Carte was produced. Like the first, though printed on sheets of uniform height the timeline proper occupies a narrow band at the Creation which widens as it approaches the author's own time. Even so the uniform timescale means that the early period is noticeably lacking in data. Priestley $(1778$, p. 21) noted for his own chart: 'I have begun the chart with David [...]. If I had begun earlier, I should have had no names for the greatest part.' This tendency inherent in all historiography reaches an alarming state in Barbeu-Dubourg's second edition, since it included the recent event avalanche of the French Revolution. At the Creation the timeline is $140 \mathrm{~mm}$ high; by the birth of Christ it is $320 \mathrm{~mm}$; in 1000AD $385 \mathrm{~mm}$; by $1600 \mathrm{AD}$ the full $428 \mathrm{~mm}$ height of the page - thus far there is no problem. But the attempt to use a uniform scale effectively collapses for the most recent period - virtual extensions to the height, presented sequentially, mean that the height for 1770 to 1800 is really $1,320 \mathrm{~mm}$, and for 1800 is $1,760 \mathrm{~mm}$ !

An additional difficulty, particularly given the totalising tendency of eighteenth century scholarship including the wish to map all time, is that in just this period time grew dramatically longer. The theories of Hutton and others pushed Creation back by many thousands, and later millions, of years. Nevertheless timelines fixing the Creation at 4004BC continued to be produced into the nineteenth century, and in the United States still are.

In digital media an obvious solution to the need for both an overview of all time and a close view of any 
chosen period is, as in our own HiT, to use two uniform scales together: a miniature chronographic of the whole dataset at the bottom of the display, with a scrollable indicator of the portion currently visible in the upper, larger view. This is an emerging practice shared by SIMILE (2009) and Continuum (André et al., 2007), though neither of these attempts to include the entire timespan of the set in the smaller pane. This step towards a conceptual disconnection between the space to be visualised and the space of the visualisation itself will be part of our proposal for ways to reconsider historic timelines.
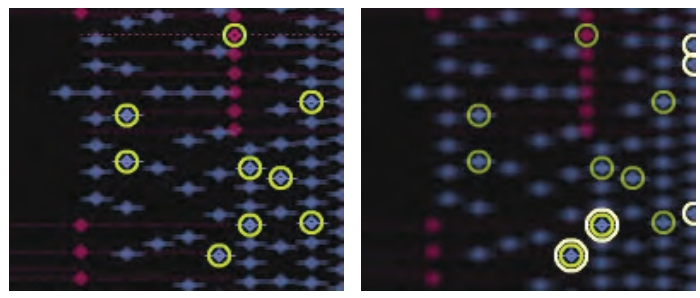

Figure 8: Bevan and Kudikov 2010: HiT for MoDA (detail). Salience for search results. Left, designs with trees are shown; right, designs with trees and fruit

\subsubsection{The problem of navigation}

When Helvicus divided history into equal pages for equal periods of time, he knew that the user could flip a handful of pages to jump to any period. Sadly, Barbeu-Dubourg's machine chronologique anticipates a problem of many digital interactive timelines: it is almost useless as a reference work, being optimised for the experiential, immersive form of engagement we noted earlier. It is intolerable to wind the crank-handles repeatedly just to look up a different period of history. Digital media of course have a solution: the user can be enabled to jump near-instantaneously from one time to another. However there is a risk associated with this: that the relationship between the timeperiod in view before the jump and that afterwards is obscured. The context-giving sense of time which is one of the main points of a chronographic approach is lost. Such loss of context is also likely to arise in visualising search results. In HiT we avoided this problem by visualising found items in the ever-present context of the timeline (Figure 8). Rather than making unwanted items disappear, we inserted a small frame or halo around items matching the most recent search. Items not selected were made recessive: with each new search, the unwanted items have their transparency and blur increased until they reach the maximum level (currently at the sixth iteration). The highlight of an item which is part of a previous search is also reduced in alpha, but has no blur applied. The 2.5D visual effect produced by this use of visual salience produces a valuable 'ghosting' or trail of previous searches. Thus the context of time (search history) as well as of space (the surrounding data) is maintained. Search results never appear outside time.

An interesting alternative to navigation between two periods is to graphically omit the intervening period, rather as a spreadsheet does when the user chooses to hide columns or rows. This approach is taken by the important Continuum project (André et al., 2007). As the authors demonstrate, this means that, for instance, the works of Bach in one century can be juxtaposed with recordings of those works in another: the intervening time is temporarily suppressed. One could say that there is an underlying linear model, but temporarily another view is created. We return to this issue below.

\subsubsection{The issue of perspective}

In his explanatory Description, Priestley reveals an interesting aspect of his approach to organising the two thousand names in the biographical chart within six bands of types of individual. He describes how, since the lower part of a large chart will generally be nearer the user's eye, he has in various instances moved the more important data to the lower, nearer part (p. 19), so, for example: 'As the Romans come in, they are made to enter by the front line, while the Greeks remove farther backwards' (1764, p. 20). These are literal cases of foregrounding, and reveal an idea akin to spatial perspective. A quasi-perspective is also employed, but in a different way, by the French chart that Priestley disapproved of. Organising time vertically it employs four timescales ranging from $22 \mathrm{~mm}=$ 500 years to $24 \mathrm{~mm}=100$ years. The effect is a kind of gradient from the most recent, fullest times, to the furthest and emptiest: the non-linear scale helps compensate for the fall-off in data in distant times. As we have seen, one of Priestley's objections to this is not the gradient per se, but the lack of indication that the scale is non-uniform. This continues to be a problem with many modern timelines whether on paper or on screen: the reasonable objective in using a non-linear scale is let down by poor visual design. Often one suspects that 'graphic design' is in fact the culprit: the desire to make a tidy, graphically uniform image actually militates against proper communication that the scale is changing. An example offering a modest solution to this problem is the Intel Technology timeline, where the bar at the bottom of the display representing the full timespan changes colour when it changes scale (Intel 2010). In HiT, with its fairly short time span, a constant timescale allowed us to avoid confronting this problem.

Discussion of perspective invites consideration of the use of three dimensions, where often the time axis is presented as depth in and out of the screen. An innovative 3D timeline of photography by Kullberg (1995) created a rich timescape of events, lifelines and associated objects that the user can 
navigate freely. Such use of 3D space for time has been evaluated by Foreman et al. (2008). While we do not have space here to discuss three dimensions more fully, such representations can offer us an important insight. In working with 3D it is clear that there is a conceptual separation between the space of the 'world' to be depicted and the space which represents it on the display, not least because the world is 3D while the display is flat. In computer graphics this is often conceived as a pipeline, from model to view to rendered image (Foley et al., 1995, pp. 334-5; 806-9); we have discussed its implications for depiction generally elsewhere (Boyd Davis, 2007). We revisit this in our third principle below.

\section{REVIEW AND FURTHER WORK}

\subsubsection{Principle 1: the value of linear timescales}

The 250-year-old arguments of Barbeu-Dubourg and Priestley for linear historic timescales still have some validity. No other rendering can give such a feel for relative intervals and durations; nor can it so accurately disclose the clusters, outliers and lacunae of history.

\subsubsection{Principle 2: the value of nonlinear timescales} So often is historiography obliged to deal with dramatically uneven densities of data, especially over extended historical periods, that it is impossible to deny the value of various non-linear scales. These include not only logarithmic scales but pragmatic adjustments of scale based on the density of data at that point (an approach often adopted in hand-made paper timelines, but also easy to implement algorithmically). Other approaches are more easily conceptualised as changes to the view, for example various kinds of virtual lens, fisheye view etc., which temporarily 'distort' the view in order to optimise the scale for the period currently of interest. We suggest this is the best way to conceive such visual manipulations.

\subsubsection{Principle 3: separating model and view}

Everyday use of digital media highlights the chameleon nature of the digital document. The same data takes on new forms at the click of a button so that, for instance in MS Word, a document may instantly appear as draft, as outline or resembling its printed form. A hierarchical structure such as an organisation chart takes on different shapes, each affording different styles of apprehension by the user. In chronographics, the omission of unwanted years in Continuum already discussed similarly exploits a conceptual separation of the underlying layout from a particular temporary view. The missing years are, as it were, still there, not just in the data but in what we might call the ideal diagram - of which only parts are visible in the real diagram. We believe such a conceptual approach can clarify our design thinking and could be of value to others.

Working with physical media, Barbeu-Dubourg and Priestley inevitably conflated their Newtonian concept of time, their ideal visual model, and the view they rendered for their users. All were linear. If instead we conceptualise discretely the data, the model, and the view, we can more easily reconcile principles 1 and 2 . We can offer users a range of controls to bend, stretch and otherwise manipulate their current view of the model (or part of it), based on their immediate needs, switching instantly between views. Digital media facilitate this separation of conceptual shape and rendered shape, because they allow the rendered shape to be contingent on the data, the user's needs and any other factors the designer wishes to take into account. Yet the design of most digital timelines ignores this potential.

\subsubsection{Principle 4: indicate scaling}

As Priestley warned, when non-linear scales are inadequately indicated, many of the advantages of chronographic visualisation are thrown away. If we are to enable users to manipulate images of time, the use and zone of influence of these tools must be patent to the user. Timelines both paper and digital routinely obfuscate the scales they use, undermining much of their potential benefit.

\subsection{Concluding remarks}

When we tackled issues in interactive digital visualisation in the HiT prototype, we were inspired by historical examples and the textual rationales that their designers had set down. We followed them in exploring the use of linear timescales and of graphical representations of uncertainty. Rather than break up our constant timescale through navigation we introduced a miniature of the entire dataset; rather than break it up when presenting search results, we adopted in-situ salience and recession. Practical development and further historical research led us to seek to combine the advantages of linear and other scales. Rather than thinking of the user simply switching between two or more views, we have adopted a conceptual separation between three things: the data, the ideal visual model (in our case rooted in the Newtonian uniform timeframe whose history we have traced in this paper) and any number of contingent views upon it. Our next step is to exploit this conceptual architecture more fully both in theory and in practice.

\section{REFERENCES}

André, P., Wilson, M.L., Russell, A., Smith, D.A., Owens, A., schraefel, m. [sic] (2007) Continuum: designing timelines for hierarchies, relationships 
and scale. In Proc. UIST. (Newport, Rhode Island, USA, 7-10 October 2007). pp. 101-110.

Barbeu-Dubourg, J. (1753) Chronographie ou Description des Temps (Chronography or Depiction of Time). Paris. Explanatory booklet for Chart, trans. Stephen Boyd Davis, October 2009. http://goo.gl/vNhN (20 March 2010).

Barbeu-Dubourg, J. (1838) Chronographie, ou Description des Temps. (Chronography or Depiction of Time). Paris. British Library 798.I.19.

Blair, J. (1754.) The chronology and history of the world, from the creation to the year of Christ, 1753.

Boyd Davis, S. (2007) A Schema for Depiction. In Van der Waarde, K. and Westendorp, P. (eds.). Visible Language 41(3). pp. 280-300.

Châtelain, H.A. (1721) Atlas Historique. Vol. 1. L'Honore \& Châtelain, Amsterdam.

Card, S.K., Mackinlay, J.D. and Shneiderman, B. (eds.) (1999) Readings in Information Visualization: using vision to think. Morgan Kaufmann.

Costigan-Eaves, P. and Macdonald-Ross, M. (1990) William Playfair (1759-1823). Statistical Science. 5(3) (August 1990). pp. 318-326.

D'Alembert, J.L-R. (1753) Chronologie. Encyclopédie ou Dictionnaire raisonné des sciences, des arts et des metiers. Vol. 3. Paris. pp. 390-400.

Diderot, D. (1753) Chronologique (machine). Encyclopédie ou Dictionnaire raisonné des sciences, des arts et des metiers. 3. Paris. 400401. Translated by Stephen Boyd Davis. The Encyclopedia of Diderot \& d'Alembert Collaborative Translation Project. University of Michigan, Ann Arbor, 2009. http://hdl.handle.net/2027/spo. did2222.0001.081 (20 March 2010).

Emerson, W. (1770) Chronology: or, the art of reckoning time. London.

Feeney, D. (2007) Caesar's Calendar: ancient time and the beginnings of history. Uni. California Press, Berkeley.

Ferguson, S. (1991) The 1753 Carte

Chronographique of Jacques Barbeu-Dubourg. Princeton University Library Chronicle. (Winter 1991). http://www.princeton.edu/ ferguson/ (20 March 2010).

Foley, J.D., van Dam, A., Feiner, S.K. and Hughes, J.F. 1995. Computer Graphics: Principles and Practice. Addison Wesley, Reading, MA: Addison Wesley.

Foreman, N., Boyd Davis, S., Moar, M., Korallo, L. and Chappell, E. (2008) Can virtual environments enhance the learning of historical chronology? Instructional Science 36 (2). pp. 155-173.
Gaukroger, S. (1995) Descartes: an intellectual biography. Clarendon Press, Oxford.

Gell, A. (1992) The Anthropology of Time: cultural constructions of temporal maps and images. Berg, Oxford.

Gould, S.J. (1987) Time's Arrow, Time's Cycle: myth and metaphor in the discovery of geological time. Harvard University Press, Cambridge MA.

Helvicus [Helwig], C. (1609) Theatrum Historicum (Historical and Chronological Theatre). Giessæ Hessorum.

Intel (2010) Intel Technology Wiki http://techwiki.intel.com/en-uk/home.aspx

Kullberg, R.L. (1995) Dynamic Timelines: visualizing historical information in three dimensions. MSc Dissertation, Massachusetts Institute of Technology, September 1995.

Locke, J. ([1693]) Some Thoughts Concerning Education. Vol. XXXVII, Part 1: Section 182. The Harvard Classics. Collier, New York, 1909-14; www.bartleby.com/37/1/ (1 August 2008).

Newton, I. (1687(1803)) Mathematical Principles of Natural Philosophy. Book 1. Scholium. Symond, 1803. Google books (15 January 2010).

Poole, R. (1998) Time's Alteration: calendar reform in early modern England. UCL Press, London.

Priestley, J. (1764) A Description of a Chart of Biography. Warrington. Available on Google Books.

Purchas, S. (1614) Purchas his pilgrimage. $2^{\text {nd }}$ edn. London. Early English books 1475-1640.

Eighteenth Century Collections Online. Gale (11 January 2010).

Rosenberg, D. (2007) Joseph Priestley and the Graphic Invention of Modern Time. Studies in Eighteenth Century Culture 36(1). pp.55-103.

Sheps, A. (1999) Joseph Priestley's Time Charts. Lumen - Proc. Representations of Time in the XVIIIth Century (Canadian Society for EighteenthCentury Studies). pp. 135-144.

SIMILE (2009) SIMILE Timeline. http://www.similewidgets.org/timeline/ (12 January 2010)

Twyman, M. (1986) Articulating Graphic Language: a historical perspective. In Wrolstad, M.E. and Fisher, D.F. Towards a New Understanding of Literacy. Praeger, New York. pp. 188-251.

Twyman, M. (1990) Textbook design: chronological tables and the use of typographic cueing. Paradigm 4 (December, 1990).

http://faculty.ed.uiuc.edu/westbury/paradigm/twyma $\underline{\text { n.html (16 January 2010) }}$ 